OPEN ACCESS

Edited by:

William Small,

Loyola University Chicago,

United States

Reviewed by:

Sushil Beriwal,

Université Pierre et Marie Curie,

France

Michael Frumovitz

University of Texas MD Anderson

Cancer Center, United States

*Correspondence:

$\mathrm{Ke} \mathrm{Hu}$

huk@pumch.cn

Fuquan Zhang

zhangfq@pumch.cn

tThese authors have contributed equally to this work

Specialty section:

This article was submitted to

Radiation Oncology,

a section of the journal

Frontiers in Oncology

Received: 02 July 2020

Accepted: 14 September 2020

Published: 02 October 2020

Citation:

Wang W, Zhou Y, Wang D, Hu K and Zhang $F$ (2020) Prophylactic Extended-Field Irradiation in Patients With Cervical Cancer: A Literature Review. Front. Oncol. 10:579410. doi: 10.3389/fonc. 2020.579410

\section{Prophylactic Extended-Field Irradiation in Patients With Cervical Cancer: A Literature Review}

\author{
Weiping Wang ${ }^{\dagger}$, Yuncan Zhou', Dunhuang Wang, Ke Hu* and Fuquan Zhang* \\ Department of Radiation Oncology, Peking Union Medical College Hospital, Chinese Academy of Medical Sciences \\ and Peking Union Medical College, Beijing, China
}

Currently, the standard radiation field for locally advanced cervical cancer patients without evidence of para-aortic lymph node (PALN) metastasis is the pelvis. Due to the low accuracy of imaging in the diagnosis of PALN metastasis and the high incidence of PALN failure after pelvic radiotherapy, prophylactic pelvic and para-aortic irradiation, also called extended-field irradiation (EFI), is performed for patients with cervical cancer. In the era of concurrent chemoradiotherapy, randomized controlled trials are limited, and whether patients with cervical cancer can benefit from prophylactic EFI is still controversial. With conformal or intensity-modulated radiation therapy, patients tolerate prophylactic EFI very well. The severe toxicities of prophylactic EFI are not significantly higher than those of pelvic radiotherapy. We recommend delivering prophylactic EFI to cervical cancer patients with common iliac lymph nodes metastasis. Clinical trials are needed to investigate whether patients with $\geq 3$ positive pelvic lymph nodes and FIGO stage IIIB disease can benefit from prophylactic EFI. According to the distribution of PALNs, it is reasonable to use the renal vein as the upper border of the radiation therapy field for patients treated with prophylactic EFI. The clinical target volume expansion of the node from the vessel should be smaller in the right para-caval region than in the left lateral para-aortic region. The right para-caval region above $\mathrm{L} 2$ or $\mathrm{L} 3$ may be omitted from the PALN target volume to reduce the dose to the duodenum. More clinical trials on prophylactic EFI in cervical cancer are needed.

Keywords: cervical cancer, prophylactic extended-field irradiation, para-aortic lymph node, radiotherapy, toxicity

\section{INTRODUCTION}

Cervical cancer is a major health problem for women in developing countries. In 2015, there were 98,900 new cases and 30,500 deaths in China (1). Para-aortic lymph node (PALN) metastasis plays an important role in the metastasis of cervical cancer. It was reported that $13.5-20.2 \%$ of patients with locally advanced cervical cancer (LACC) had positive PALNs (2-5). For patients with FIGO stage IB, IIB and III disease, the incidences of PALN metastasis are 5\%, 16\% and 25\% (4). According to the 2018 FIGO staging system, patients with positive PALNs are allocated to the stage IIIC2 category (6).

At present, the standard treatment for patients with LACC is concurrent chemoradiotherapy. For patients with positive PALNs, extended-field irradiation (EFI) of the pelvis and paraaortic region is recommended. Extended-field intensity-modulated radiation therapy (IMRT) combined with brachytherapy and concurrent chemotherapy is safe and effective for patients with positive PALNs $(7,8)$. For patients without evidence of positive 
PALNs, the standard treatment target volume is the pelvis, and the PALN region is not included.

With image-guided brachytherapy, the failure pattern of patients with cervical cancer has changed. And PALN failure is a main failure site. An analysis from the retroEMBRACE study reported that, after image-guided brachytherapy, the pelvic failure, PALN failure, systemic failure and distant (systemic + PALN) failure accounted for 13, 9, 21, and $24 \%$ of failure, respectively (9). Another study with EMBRACE study cohort found that, positive LNs at diagnosis are mainly located in the pelvis. However, LNs failure are more often in PALN region. Of patients with nodal failure, 39\% were located in PALN region (10). Huang et al. (11) reported that $11 \%$ of patients with cervical cancer experienced PALN recurrence after pelvic radiotherapy. In patients with positive pelvic lymph nodes (LNs), the 5-year PALN recurrence rate was $37 \%$. For patients without positive PALNs on imaging, prophylactic EFI has the potential to reduce the incidence of PALN treatment failure after definitive radiotherapy. Currently, whether patients with LACC can benefit from prophylactic EFI is still controversial. In the present article, we review the literature and discuss the necessity, indications, toxicities and target volume contouring of prophylactic EFI in patients with cervical cancer.

\section{PET/CT IN DETECTING PALN METASTASIS}

According to the 2018 FIGO staging system, positive PALNs can be diagnosed with imaging or pathology (6). At present, there is insufficient evidence that surgical staging is beneficial for patients with LACC (12-16). PALN status is determined by imaging for most patients. In meta-analysis, the pooled sensitivities of CT, MRI, and PET/CT were 68, 54, and 71-81\%, respectively, in detecting PALN in patients with cervical cancer, and the corresponding specificities were 90,94 , and $97-98 \%$, respectively $(17,18)$. The positive predictive value and negative predictive value of PET/CT in detecting PALN were 50-100 and $83-100 \%$, respectively (18).

PET/CT has better sensitivity and specificity than CT and MRI in detecting PALN. However, the accuracy of PET/CT in the diagnosis of PALN metastasis is not sufficient, especially for patients with positive LNs. For PET/CT, it was reported that the false negative rate of PALN metastasis was $8.4-13.6 \%$ in patients with LACC $(19,20)$, which is comparatively low. However, in patients with positive pelvic LNs on PET/CT, the false negative rate was $24-27.7 \%(19,20)$, and the negative predictive value of PET/CT in diagnosing PALN metastasis was only $77.6 \%$ (5). Ramirez et al. (21) reported that $22 \%$ patients with positive pelvic but negative para-aortic LNs on PET/CT had positive histologically confirmed positive PALN.

De Cuypere et al. (5) reported that in patients with LACC, $20.2 \%$ had histologic positive PALNs, $10.1 \%$ had positive PALNs on PET/CT and $4.8 \%$ had positive PALNs both on PET/CT and surgical staging. In patients with positive pelvic LNs on PET/CT, $27.7 \%$ had histologic positive PALNs, $19.3 \%$ had positive PALNs on PET/CT and $9.6 \%$ had positive PALNs both on PET/CT and surgical staging. Imaging modalities, including PET/CT, definitely underestimate the PALN metastasis rate.

The accuracy of PET/CT in the diagnosis of PALN metastasis is not sufficient, and PET/CT underestimate the PALN metastasis rate, especially for patients with positive pelvic LNs.

\section{PROPHYLACTIC EFI WITHOUT CONCURRENT CHEMOTHERAPY}

Before concurrent chemotherapy became the standard treatment, most patients with LACC received definitive radiotherapy alone. Three randomized controlled trials (RCTs) compared the efficacy and toxicities of pelvic radiotherapy and prophylactic EFI in patients with cervical cancer. In RTOG 79-20,367 patients with FIGO stage IB or IIA (primary tumor $\geq 4 \mathrm{~cm}$ ) or with FIGO stage IIB cervical cancer were randomized to the pelvic-only irradiation and prophylactic EFI arms. Conventional radiotherapy was used. In the pelvic-only irradiation and prophylactic EFI arms, the 10-year overall survival (OS) rates were 44 and $55 \%(p=0.02)$, respectively, the disease-free survival rates were 40 and $42 \%(p=0.44)$, respectively, and the cumulative incidences of first distant failure were 16 and $23 \%$ ( $p=0.053)$, respectively. Prophylactic EFI tended to increase grade 4-5 toxicities and the death rate due to radiotherapy (22). The other two trials revealed that patients with cervical cancer did not benefit from prophylactic EFI. In the RCT of the EORTC radiotherapy group, compared with pelvic radiotherapy (228 patients), prophylactic EFI (213 patients) did not improve DFS or distant failure and increased the incidence of severe digestive complications (23). An RCT from Japan also revealed that prophylactic EFI did not improve cause-specific survival and distant failure and was associated with more late complications (24). A Cochrane meta-analysis included the three RCTs mentioned above and found that EFI reduced the risk of death (hazard ratio, HR 0.67, 95\% confidence interval, CI 0.480.94 ) and PALN recurrence (risk ratio 0.36, 95\% CI 0.18-0.70) and that the risk of disease progression was not significantly improved (25).

From 1999-2000, several clinical trials demonstrated that, compared with radiotherapy alone, cisplatin-based concurrent chemoradiotherapy improved the survival of patients with cervical cancer (26-29). Thus, LACC treatment entered the era of concurrent chemoradiotherapy. RTOG 90-01 revealed that pelvic radiotherapy combined with concurrent chemotherapy resulted in OS and DFS rates that were superior to those of prophylactic EFI without concurrent chemotherapy in patients with cervical cancer $(30,31)$. Clinical trials on prophylactic EFI then stagnated for years.

\section{PROPHYLACTIC EFI COMBINED WITH CONCURRENT CHEMOTHERAPY}

In the era of concurrent chemoradiotherapy, clinical trials comparing prophylactic EFI and pelvic radiotherapy have been limited. In the study by Asiri et al. (32), 102 patients 
with FIGO stage IIB-IVA cervical cancer and no evidence of PALN metastasis were randomly assigned to the pelvis concurrent chemoradiotherapy arm (50 patients) and extended-field concurrent chemoradiotherapy arm (52 patients). Seventy-four patients were analyzed. In the pelvic concurrent chemoradiotherapy and extended-field concurrent chemoradiotherapy arms, the PALN control rates were 82.1 and $97.1 \%(p=0.02)$, respectively, the distant control rates were 74.7 and $86.9 \%(p=0.03)$, respectively, the DFS rates were 69.1 and $80.3 \%(p=0.03)$, respectively, and the OS rates were 60.4 and $72.4 \%(p=0.04)$, respectively (32). This is the only published RCT (25). Based on the small sample size, imbalance of allocation, and different radiotherapy techniques between the two groups, the outcome of this trial should be interpreted with caution. In the Cochrane metaanalysis, this study was evaluated as having a "high risk of bias" (25).

Some other retrospective studies have compared prophylactic EFI combined with concurrent chemotherapy to pelvic radiotherapy. Lee et al. (33) analyzed 206 LACC patients with negative PALNs. Of these patients, 110 and 96 underwent pelvic radiotherapy and prophylactic EFI, respectively. The upper border of the prophylactic EFI field was at the level of the left renal vessel. In the pelvic radiotherapy and prophylactic EFI patient groups, the 5-year PALN recurrence-free survival rates were 87.6 and $97.9 \%(p=0.03)$, respectively, and the OS rates were 74.5 and $87.8 \%(p=0.04)$, respectively (33). A retrospective study from our institute included 778 cervical cancer patients without evidence of PALN metastasis. Of these patients, 154 were treated with prophylactic EFI, 624 received pelvic radiotherapy, and $83 \%$ received concurrent chemotherapy. After multivariate analysis, prophylactic EFI was an independent prognostic factor of distant failure and PALN failure and not an independent factor of OS or DFS. After propensity score matching, patients treated with prophylactic EFI experienced less distant failure ( 7.0 vs $21.7 \%, p=0.016$ ) and PALN failure ( 0 vs $6.6 \%, p=0.014$ ) than patients who received pelvic radiotherapy. However, OS and DFS were not significantly different between the two groups (34). In retrospective studies conducted by $\mathrm{Yi}$ et al. (35), Oh et al. (36), Park et al. (37), and Yap et al. (38), patients with cervical cancer did not benefit from prophylactic EFI. In the study by Park et al. (37), 203 patients with LACC were included. Prophylactic EFI and pelvic radiotherapy were performed in 88 and 115 patients, respectively. In the pelvic radiotherapy and prophylactic EFI patient groups, the 5-year OS rates were 74.8 and $71.7 \%(p=0.699)$, respectively, and the DFS rates were 74.5 and $75.8 \%(p=0.668)$, respectively (37). It should be noted that in most retrospective studies, the baseline characteristics were not balanced between the two groups. Patients in the prophylactic EFI group tended to have more advanced disease. For example, in the study by Park et al. (37), more patients in the prophylactic EFI group had positive pelvic LNs ( 60.7 vs $29.6 \%, p<0.001$ ), large primary tumors (67.1 vs $42.6 \%, p=0.002$ ), and more advanced stages (FIGO stage IIIB: 27.3 vs $13.0 \%, p=0.019)$ than those in the pelvic radiotherapy group. With this unbalanced baseline, the studies had a high risk of bias.
At present, several clinical trials comparing prophylactic extended-field concurrent chemotherapy and pelvic concurrent chemoradiotherapy have been registered. NCT 00980759/KROG 07-01 is a phase 2 RCT from South Korea. Cervical cancer patients with negative PALNs were randomly assigned to the EFI arm (para-aortic and pelvic irradiation with chemotherapy) and pelvic radiation therapy arm (pelvic irradiation with chemotherapy). The trial started in 2006, and the estimated enrolment number was 312 patients (39). In part 1 of the study, patients were enrolled according to the status of CA9 (hypoxia marker) expression. In part 1, the investigators found that prophylactic EFI reduced PALN recurrence in patients with CA9-positive tumors but that the survival outcome was not improved (40). The final results of the trial have not been released. In 2019, our institute started a multicentre, randomized, phase 3 trial comparing pelvic radiotherapy to prophylactic EFI in selected patients with cervical cancer treated with concurrent chemoradiotherapy in China (NCT03955367) (41). Three other clinical trials, including the NCT 01063387 trial (42), ChiCTRIPR-14005499 trial (43) and ChiCTR-IIR-17013683 trial (44), were registered. The EMBRACE II study is a prospective study with multiple interventions and multiple endpoints. In this study, nodal clinical target volume (CTV) is defined according to the risk of nodal spread. Patients in the high-risk LNs group will receive pelvis and PALN region irradiation (45).

In the era of concurrent chemoradiotherapy, RCTs are limited, and whether patients with cervical cancer can benefit from prophylactic EFI is still controversial. Thus, more clinical trials addressing prophylactic EFI are needed.

\section{THE TOXICITIES OF EFI}

With conventional radiotherapy, the toxicity of prophylactic EFI is unacceptable and much higher than that of pelvic radiotherapy. In the prophylactic EFI and pelvic radiotherapy groups of RTOG 79-20, the incidences of 10-year grade 4-5 toxicities from radiotherapy were 8 and $4 \%(p=0.06)$, respectively, and the mortality rates due to radiotherapy complications were 2 and $1 \%(p=0.24)$, respectively (22). In the trial of the EORTC radiotherapy group, the 4-year grade 3-4 digestive complications were 8.0 and $3.5 \%(p=0.005)$ in the prophylactic EFI and pelvic radiotherapy groups, respectively (23). In the trial from Japan, complications were more frequent in the prophylactic EFI group than in the pelvic radiotherapy group $(13 / 45$ vs $2 / 48, p<0.025)$ (24). In RTOG 01-16 and RTOG 92-10, EFI was delivered to patients with para-aortic or common iliac LN metastasis with conventional radiotherapy, and the incidence of late grade 34 toxicities was $24-40 \%(7,46)$. Based on the high incidence of toxicities, delivery of prophylactic EFI with conventional radiotherapy is not recommended.

In recent years, conformal radiation therapy and IMRT have been used in prophylactic EFI for patients with cervical cancer. In part 1 of NCT 00980759/KROG 07-01, 116 patients were randomized to the prophylactic EFI group or pelvic radiotherapy group. Radiotherapy was delivered with conformal radiation therapy. The acute and late toxicities of patients in 
the prophylactic EFI group and EFI group were not significantly different. In the prophylactic EFI group, the incidences of grade 3-4 acute upper gastrointestinal, lower gastrointestinal, late gastrointestinal and genitourinary toxicities were $0,5.3,3.5$, and $1.8 \%$, respectively (40). In the RCT of Asiri et al. (32), radiotherapy was performed with conformal radiation therapy or IMRT. The acute and late toxicities were similar between the pelvic radiotherapy and prophylactic EFI groups. In the study by Lee et al. (47), radiotherapy was delivered with IMRT. The incidences of late $\geq$ grade 3 toxicities in the prophylactic EFI and pelvic radiotherapy groups were not significantly different (3.8 and $2.5 \%$ ), and no $\geq$ grade 3 genitourinary toxicity was observed in either group. Furthermore, the incidences of acute $\geq$ grade 3 toxicities were also similar between the two groups (47). In our study, the incidences of $\geq$ grade 3 late toxicities were 6.5 and $3.5 \%$ in the prophylactic EFI and pelvic radiotherapy groups, respectively ( $p=0.097)$ (34). With conformal or IMRT, patients tolerate prophylactic EFI very well. The incidence of severe toxicities from prophylactic EFI is not significantly higher than that from pelvic radiotherapy.

Several prospective randomized trials and one meta-analysis demonstrated that the toxicities of IMRT were lower than that of conformal radiation therapy in patients with LACC treated with definitive radiation therapy (48-51). In the study by Gandhi et al. (51), 44 patients with 2009 FIGO stage IIBIIIB cervical cancer were randomized to receive whole pelvic conventional radiation therapy or IMRT. Patients in the IMRT arm experienced fewer $\geq$ grade 2 acute gastrointestinal toxicities (31.8 vs $63.6 \%, p=0.034$ ) and $\geq$ grade 3 gastrointestinal toxicities ( 4.5 vs $27.3 \%, p=0.047$ ) compared with patients in the conformal radiotherapy arm (51). A meta-analysis included 6 studies encompassing 1008 patients with cervical cancer who received definitive radiotherapy. Of them, 350 patients treated with IMRT and 658 patients received two-dimensional radiotherapy or three-dimensional conformal radiotherapy. The OS and DFS were not significantly different between the two groups. IMRT significantly reduced $\geq$ grade 3 acute gastrointestinal toxicities (odds ratio, OR, $0.55,95 \%$ CI $0.32-0.95, p=0.03$ ), $\geq$ grade 3 acute genitourinary toxicities (OR $0.31,95 \%$ CI $0.14-0.67, p=0.003$ ), and $\geq$ grade 3 chronic genitourinary toxicities (OR $0.09,95 \% \mathrm{CI}$ $0.01-0.67, p=0.02$ ). In the ASTRO clinical practice guideline of 2020, IMRT is conditionally recommended to decrease acute and chronic toxicity in definitive radiotherapy for cervical cancer (52). It should be noted that most patients in these studies are treated with pelvic radiotherapy. However, given the larger target volume and additional organs at risks, IMRT is likely to decrease the risk of toxicities of prophylactic EFI compared with conformal radiation therapy.

\section{THE INDICATIONS OF PROPHYLACTIC EFI}

The eligibility criteria differ between studies on prophylactic EFI, and the criteria include cervical cancer $(33,35)$, LACC $(37,40)$, FIGO stage IB-IVA (34), FIGO stage IIB-IVA (32), and pelvic $\mathrm{LN}$ involvement $(36,40,47)$, among others. This is an important reason why these studies have contradictory results. The survival of patients with early-stage cervical cancer is high, with 3-year OS and DFS rates higher than $90 \%$ after concurrent chemoradiotherapy (53). For patients with early-stage disease, it is difficult to improve survival with prophylactic EFI. Due to the toxicities of EFI, investigating who could benefit from prophylactic EFI is the main goal now.

Due to the low sensitivity of imaging modalities $(17,18,54)$, cervical cancer patients with negative PALNs on imaging may have occult PALN metastasis before treatment. If EFI is not conducted, patients with occult PALN metastasis will experience PALN failure after treatment. Therefore, patients with a high risk of PALN metastasis may benefit from prophylactic EFI. Shim et al. (3) analyzed 245 patients with LACC who underwent paraaortic lymphadenectomy. Of these patient, 34 had pathologically proven positive PALNs. After multivariate analysis, PALN status on PET/CT and tumor size on magnetic resonance imaging were independent predictors of PALN metastasis. A scoring system to estimate PALN status was constructed. PALN metastasis on PET/CT was scored as 5 and 0 for yes and no. Tumor size $\leq 4 \mathrm{~cm}, 4.01-5 \mathrm{~cm}$, and $>5 \mathrm{~cm}$ were scored as 0,1 , and 3 points, respectively. For patients at low (0-1 points), intermediate ( 3 points) and high risk ( $=5$ points) of PALN metastasis, the predicted probabilities of PALN metastasis were $2.9,20.8$, and $76.2 \%$, respectively (3). Han et al. (55) analyzed 723 patients with FIGO stage IB1-IIA2 cervical cancer who were treated with radical hysterectomy/radical trachelectomy, pelvic LN dissection, and PALN dissection. They found that PALN metastasis was associated with age $>46$ years, primary tumor size $>3.5 \mathrm{~cm}$ and FIGO stage IIA (55). In our study based on imaging, PALN metastasis was associated with histology, tumor size, bilateral pelvic LN metastasis and common iliac LN metastasis (56).

As prophylactic EFI reduces PALN recurrence in patients with cervical cancer $(32,34,47)$, patients with a high risk of PALN recurrence may benefit from prophylactic EFI. Huang et al. (11) analyzed 758 patients with cervical cancer who were treated with pelvic radiotherapy. After a median followup of 50 months, 80 patients had PALN recurrences. In a multivariate analysis, squamous cell carcinoma (SCC) antigen level $>40 \mathrm{ng} / \mathrm{ml}$, advanced parametrial involvement and pelvic lymphadenopathy were independent factors associated with PALN recurrence. For patients with SCC antigen $>40 \mathrm{ng} / \mathrm{ml}$, advanced parametrial involvement, pelvic lymphadenopathy and no risk factors, the 5-year PALN recurrence rates were 57, 34, 37, and $9 \%$, respectively (11).

The PALN region plays an important role in the distant metastasis of cervical cancer, especially in mediastinal and supraclavicular LN metastasis. Prophylactic EFI may block the pathway of para-aortic lymphatic metastasis and can reduce distant failure in patients with cervical cancer $(32,34,57)$. In a previous study, we analyzed 797 cervical cancer patients treated with concurrent chemoradiotherapy and found that non-SCC, common iliac LN metastasis and bilateral pelvic LN metastasis were independent predictors of distant failure. For patients with high-risk (2-3 risk factors) and low-risk (0-1 risk factors) distant failure, the distant failure rates were $39.3 \%$ and $19.3 \%(p<0.001)$, 
respectively (58). It was reported that positive pelvic LNs (5961 ), advanced FIGO stage (61) and SCC antigen (60) are also associated with distant failure.

In the discussions above, we find that a large primary tumor, advanced FIGO stage, pelvic LN metastasis, bilateral pelvic LN metastasis, common iliac LN metastasis, non-SCC and high SCC antigen levels are risk factors for PALN metastasis before treatment or PALN recurrence and distant failure after treatment. The factors may be used as potential indications of prophylactic EFI. However, this is indirect evidence. These identified risk factors should be validated to determine whether they are indeed predictive of the therapeutic success of prophylactic EFI in patients with cervical cancer. At present, direct evidence on the indications of prophylactic EFI is limited. In the study comparing prophylactic EFI to pelvic radiotherapy by Lee et al. (33), the subgroup analysis revealed that in the pelvic radiotherapy and prophylactic EFI groups, the patients with FIGO III-IVA disease or positive pelvic LNs had 5-year PALN recurrence-free survival rates of 80.1 and $96.4 \%(p=0.02)$, respectively, and OS rates of 58.1 vs $83.5 \%$, $(p=0.012)$, respectively. However, these outcomes were not significantly different for patients with FIGO IB-IIB and negative pelvic LNs (33). Another study by Lee et al. (47) analyzed 198 cervical cancer patients with positive pelvic LNs and negative PALN. In the pelvic radiotherapy and prophylactic EFI groups, the patients with positive common iliac LNs or $\geq 3$ positive pelvic LNs had 5-year PALN recurrence-free survival rates of 56.5 and 93.9\% ( $p<0.001)$, respectively, and cancer-specific survival rates of 56.8 and $100 \%(p<0.001)$, respectively. These outcomes were not significantly different in patients with positive pelvic LNs below the common iliac bifurcation and 1-2 pelvic LNs (47). A study from our institute analyzed 133 patients with 2018 FIGO stage IIIB cervical cancer who were treated with concurrent chemoradiotherapy or radiotherapy. In the pelvic radiotherapy and prophylactic EFI patient groups, the 3-year OS rates were 66.3 and $80.3 \%(p=0.013)$, respectively, and the DFS rates were 57.2 and $80.4 \%(p=0.002)$, respectively $(62)$.

As presented above, common iliac LN metastasis is associated with PALN metastasis before treatment and distant failure after concurrent chemoradiotherapy $(56,58)$. We have direct evidence that patients with common iliac LN metastasis can benefit from prophylactic EFI (47). In the EMBRACE II study, patients with a positive LNs at the common iliac will be grouped into the high-risk LNs group and will receive EFI (45). In the NCCN guidelines (63) and the review by Jurgenliemk-Schulz et al. (64), EFI is recommended for patients with positive common iliac LNs. Therefore, common iliac LN metastasis is a clear indication of prophylactic EFI. Due to ethical issues, patients with common iliac LN metastasis were excluded from the ChiCTR-IIR-17013683 trial, ChiCTR-IPR-14005499 trial and NCT03955367 trial $(41,43,44)$. It has been reported that pelvic LN metastasis is associated with PALN metastasis, PALN recurrence and distant failure $(11,56,59-61)$. A retrospective study indicated that prophylactic EFI improved the survival of patients with positive pelvic LNs (33), and thus, patients with positive pelvic LNs may benefit from prophylactic EFI. Because bilateral pelvic LN metastasis is reported to be an independent risk factor of distant failure (58) and because Lee et al. (47) reported that patients with 1-2 positive pelvic LNs below the common iliac bifurcation do not benefit from prophylactic EFI, we recommend that prophylactic EFI can be delivered to patients with $\geq 3$ positive pelvic LNs in clinical trials. In the review by Jurgenliemk-Schulz et al. (64), the authors recommended that prophylactic EFI can be conducted especially for patients with more than two involved LNs. In the EMBRACE II study, > 3 positive pelvic LNs serve as an indication of EFI (45). Multiple positive pelvic LNs was also used as an inclusion criterion in the ChiCTR-IPR-14005499 trial (43) and NCT03955367 trial (41). Advanced FIGO stage and parametrial involvement were risk factors for PALN metastasis, PALN failure or distant failure (11, $55,61)$. Moreover, prophylactic EFI could improve the survival of patients with 2018 FIGO stage IIIB disease (62). Patients with stage IIIB were included in the NCT00980759, ChiCTR-IIR17013683, and NCT03955367 trials. Therefore, prophylactic EFI can be delivered to patients with FIGO stage IIIB in clinical trials. A large primary tumor, non-SCC and high SCC antigen were predictors of a high PALN metastasis rate, PALN recurrence and distant failure $(3,55,56,58,60)$. However, these factors were also associated with worse local control $(61,65,66)$. Prophylactic EFI cannot improve the survival of patients with local failure. We do not have direct evidence that patients with these three factors can benefit from prophylactic EFI. Therefore, a large primary tumor, non-SCC and high SCC antigen levels are not recommended as indications of prophylactic EFI.

We recommend delivering prophylactic EFI to cervical cancer patients with common iliac LN metastasis. Clinical trials are needed to investigate whether patients with $\geq 3$ positive pelvic LNs, and FIGO stage IIIB disease can benefit from prophylactic EFI.

\section{DELINEATION AND DOSE PRESCRIPTION OF THE PALN REGION}

Currently, the delineation of the PALN region is inclusive. The delineation of clinical target volume (CTV) is based on the distribution of PALNs. Several studies on the distribution of PALNs are summarized in Table 1.

Generally, the PALN region refers to the area adjacent to the aorta and inferior vena cava from the aortic bifurcation to T12. In the study of Keenan et al. (67), all PALNs were inferior to the left renal vein. Takiar et al. (68) reported that 96\% of PALNs were in the inferior and middle third of the PALN region. In the retrospective study by Lee et al. (33), 96 patients received prophylactic sub-renal vein radiotherapy. Only 3 patients (3.1\%) experienced PALN failure (34). A smaller CTV results in decreased toxicities. As the incidence of PALN metastasis above the renal vein is low and PALN failure is rare after prophylactic sub-renal vein radiotherapy, it is reasonable to use the renal vein as the upper border of the radiation therapy field for patients treated with prophylactic EFI. In the EMBRACE II study and the review of Jurgenliemk-Schulz et al. (64), the level of renal veins is also used or recommended as the upper border of CTV in patients treated with EFI (45). 
TABLE 1 | Studies on the distribution of para-aortic lymph nodes in patients with cervical cancer.

\begin{tabular}{|c|c|c|c|c|c|c|c|c|c|}
\hline \multirow[t]{2}{*}{ Authors [ref] } & \multirow[t]{2}{*}{$\begin{array}{l}\text { Lymph node } \\
\text { diagnostic } \\
\text { modalities }\end{array}$} & \multirow[t]{2}{*}{$\begin{array}{l}\text { No. of patients } \\
\text { (positive PALNs) }\end{array}$} & \multicolumn{3}{|c|}{$\begin{array}{c}\text { Mean distance from lymph node } \\
\text { to adjacent anatomic } \\
\text { structures }\end{array}$} & \multicolumn{3}{|c|}{ Distribution of PALNs } & \multirow[b]{2}{*}{$\begin{array}{c}\text { SI } \\
\text { direction }\end{array}$} \\
\hline & & & LLPA & AC & PRC & LLPA & AC & PRC & \\
\hline $\begin{array}{l}\text { Keenan et al. } \\
(67)\end{array}$ & PET/CT & $\begin{array}{l}\text { Design cohort: } 21 \\
\text { (39); validation } \\
\text { cohort: } 10(29)\end{array}$ & $\begin{array}{c}8 \mathrm{~mm} \text { from } \\
\text { aorta }\end{array}$ & $\begin{array}{c}8 \mathrm{~mm} \text { from } \\
\text { aorta, } \\
6 \mathrm{~mm} \text { from } \\
\text { IVC }\end{array}$ & $\begin{array}{c}5 \mathrm{~mm} \text { from } \\
\text { IVC }\end{array}$ & $49 \%$ & $46 \%$ & $5 \%$ & $\begin{array}{l}\text { All PALNs } \\
\text { were } \\
\text { inferior to } \\
\text { the left } \\
\text { renal vein. }\end{array}$ \\
\hline Takiar et al. (68) & $\mathrm{PET} / \mathrm{CT}$ & $30(72)$ & $8.3 r$ & from aorta & & $51 \%$ & $44 \%$ & $4 \%$ & $\begin{array}{c}\text { Inferior third } \\
60 \% \text {; } \\
\text { Middle third } \\
36 \% \text {; Up } \\
\text { third 3\%. }\end{array}$ \\
\hline
\end{tabular}

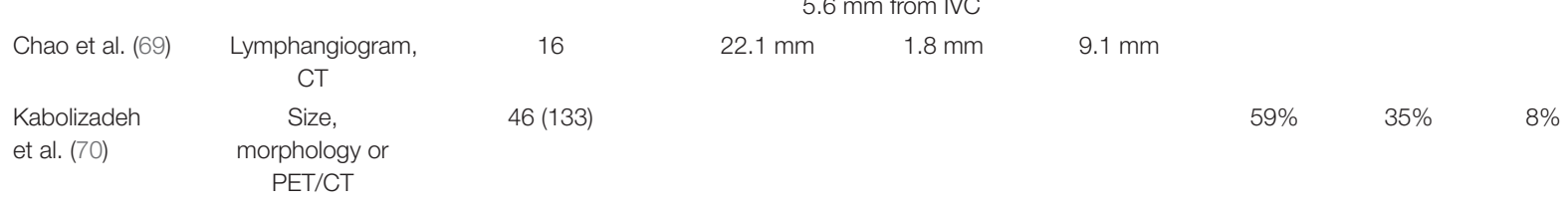

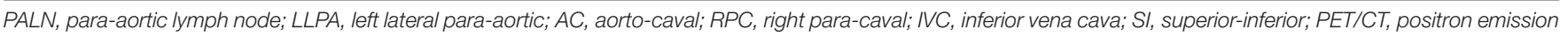
tomography/computed tomography.

PALNs are classified as left lateral para-aortic (LLPA), aortocaval (AC), and right para-caval (PRC) PALNs based on their location. As shown in Table 1, the mean distances from the LNs to adjacent anatomic structures are $8-22.1 \mathrm{~mm}$ in the LLPA region, 1.8-8 $\mathrm{mm}$ in the AC region and 5-9.1 $\mathrm{mm}$ in the PRC region (6769). Most PALNs are located in the LLPA and AC regions. Only 4$6 \%$ of PALNs are located in the PRC region $(67,68,70)$. As LNs in the $\mathrm{PRC}$ region are rare and the mean distance from the inferior vena cava to LNs in the PRC region is small, it is reasonable that the node CTV expansion from vessels can be small in the PRC region. Keenan et al. (67) recommended a node CTV expansion of $10 \mathrm{~mm}$ circumferentially and $15 \mathrm{~mm}$ laterally from the aorta and of $8 \mathrm{~mm}$ anteromedially and $6 \mathrm{~mm}$ posterolaterally from the inferior vena cava to cover $97 \%$ of PALNs. In the study of Chao et al. (69), to cover $100 \%$ of lymphangiography-aid PALNs, the node CTV comprised the aorta plus $2 \mathrm{~cm}$ and inferior vena cava plus $1 \mathrm{~cm}$ and the region $5 \mathrm{~mm}$ ventral to the aorta. In addition, PRC LNs are rare above L2 or L3. Takiar et al. (68) reported that all PRC LNs were limited to the inferior third of the paraaortic region (below L3). Keenan et al. (67) found that no PRC lymph node was located above L2 and recommended excluding the RPC region above the L1-L2 interspace. Therefore, the RPC region above L2 or L3 may potentially be omitted from the PALN target volume if the PALN region above L2 or L3 is irradiated. This significantly reduces the dose to the duodenum (71) and may further result in a lower incidence of duodenal toxicities when conducting prophylactic EFI.

In studies which prophylactic EFI was delivered with IMRT or conformal radiation therapy, the dose/fractionation schedules used included 45 Gy in 25 fractions $(35,40,45), 50.4$ Gy in 28 fractions $(33,34)$, and $45-50.4$ Gy in $25-28$ fractions $(32,41,47)$. NCCN guidelines recommended that coverage of microscopic nodal metastasis requires an EBRT dose of approximately $45 \mathrm{~Gy}$ (63). Given that studies comparing different dose/fractionation schedules in prophylactic EFI are lacking, the same dose/fractionation schedule for pelvic nodal coverage (45-50.4 Gy in 25-28 factions) can be used in patients with prophylactic EFI.

\section{SURGICAL STAGING OF PALN REGION}

Surgical staging and pathologic assessment can provide precise information on PALN status. Given that the accuracy of PET/CT in detecting PALN metastasis remains unsatisfactory $(17,19,20)$, surgical staging still has an important role in the era of PET/CT in PALN status assessment.

Compared with imaging staging based CCRT, surgical staging allows accurate adaptation of radiotherapy field. Uterus-11 is a multicenter phase III trial comparing surgical staging followed by CCRT (arm A) and clinical staging followed by CCRT (arm B). A total of 240 patients were eligible, with 121 patients in arm A and 119 patients in arm B. Patients with PALN involvement underwent EFI. Surgical staging resulted in more patients undergoing EFI (23\% in arm A vs $12 \%$ in arm B, $p<0.014)(16)$. In Uterus-11, surgical staging led to upstaging in $33 \%$ of patients (72). De Cuypere et al. (5) reviewed 168 patients with LACC underwent pretreatment PET/CT and PALN dissection. Of them, 35 patients $(20.8 \%)$ underwent radiotherapy field adaption after PALN dissection. For patients with pelvic LN involvement on PET/CT, 27.7\% had radiotherapy field modification. In the study of Gonzalez-Benitez et al. (14), PALN status were assessed with imaging in 31 patients and with surgical staging in 43 patients. In imaging and surgical staging group, EFI was performed in 19.4 
and $44.2 \%$ of patients (14). In the study of De Cuypere et al. (5) $15(18.1 \%)$ of 83 patients with positive pelvic LNs changed the target volume from the pelvis to the pelvis and PALN region after surgical staging. Ramirez reported that $18.3 \%$ of patients had a treatment modification based on surgical findings (21). Mezquita et al. (73) reported that $13.4 \%$ of patients had treatment modification to the para-aortic area after surgical staging.

A drawback of surgical staging is the delay of CCRT $(13,74)$. In Uterus-11, the mean time interval between surgical staging and the beginning of CCRT was 13 days (7-21 days) (72). In the study of Becker et al. (75), the average delay between surgery staging and CCRT was 22 days. Yang et al. (13) reported that the median time duration from diagnosis of cervical cancer to CCRT was 47 days and 28 days in patients treated with surgical staging followed by CCRT and CCRT $(p<0.01)$.

The survival benefit of surgical staging in patients with LACC is still controversial. Lai et al. (12) conducted a randomized trial comparing clinical staging (arm A, $n=29$ ) and surgical staging ( $\operatorname{arm~B,} n=32$ ) in patients with LACC. Patients receive definitive CCRT or radiotherapy after staging. Patients in arm B had worse PFS $(p=0.003)$ and OS $(p=0.024)$ compared with patients in arm A. The HR of relapse/persistent disease and death ( $\operatorname{arm~B}$ vs arm A) were $1.71(p=0.006)$ and 1.50 $(p=0.028)$, respectively. The toxicities between two groups were not significantly different. This trial was stopped after an interim analysis because of the survival reduction of patients in arm B (12). Yang et al. (13) retrospectively analyzed 148 patients with FIGO stage IB2-IIIB cervical cancer treated with CCRT. With propensity score-matching (1:2), 35 patients in the surgical staging group and 70 patients in the imaging group were selected. In surgical staging and imaging groups, the 5-year PFS were 62.6 and $72.4 \%(p=0.77)$, the 5-year OS were 70.2 and $70.5 \%(p=0.96)$, respectively (13). In the study of Gonzalez-Benitez et al. (14), the mean PFS and OS were not significantly different between patients treated with surgical staging and imaging staging. Gold et al. (15) analyzed 555 patients underwent PALN surgical staging and 130 patients underwent radiographic evaluation from GOG 85, GOG 120 and GOG 165. In patients with stage III-IV disease, the 4year PFS were 48.9 and $36.3 \%$, and the OS were 54.3 and $40 \%$ in surgical staging and radiographic groups, respectively. After multivariate analysis, surgical staging was associated with better PFS ( $p=0.043$ ) (15). The survival outcome of Uterus11, a multicenter phase III trial comparing surgical staging followed by CCRT and clinical staging based CCRT, has not been reported (16).

\section{REFERENCES}

1. Chen W, Zheng R, Baade PD, Zhang S, Zeng H, Bray F, et al. Cancer statistics in China, 2015. CA Cancer J Clin. (2016) 66:115-32. doi: 10.3322/caac.21338

2. Vazquez-Vicente D, Fernandez Del Bas B, Garcia Villayzan J, Di Fiore HA, Luna Tirado J, Casado Echarren V, et al. Laparoscopic paraaortic surgical staging in locally advanced cervical cancer: a single-center experience. Clin Transl Oncol. (2018) 20:1455-9. doi: 10.1007/s12094-018-1878-4

3. Shim SH, Kim DY, Lee SJ, Kim SN, Kang SB, Lee SW, et al. Prediction model for para-aortic lymph node metastasis in patients with locally advanced
It should be noted that, only patients with positive PALN received EFI in these studies $(12,16,76)$, which means that prophylactic EFI was not conducted in patients in imaging group. To our knowledge, prospective studies comparing surgical staging followed by tailored radiotherapy and imaging staging based prophylactic EFI are lacking at present.

Compared with imaging staging based CCRT, surgical staging allows accurate adaptation of radiotherapy field, and leads to the delay of CCRT. It is still controversial whether surgical staging could improve the survival of patients with LACC. And prospective studies comparing surgical staging followed by tailored radiotherapy and imaging staging based prophylactic EFI are lacking at present.

\section{CONCLUSION}

It is still controversial whether patients with cervical cancer could benefit from prophylactic EFI. With conformal radiation therapy or IMRT, prophylactic EFI is well tolerated. We recommend delivering prophylactic EFI to cervical cancer patients with common iliac LN metastasis. Clinical trials are needed to investigate whether patients with $\geq 3$ positive pelvic LNs, and FIGO stage IIIB disease can benefit from prophylactic EFI. According to the distribution of PALNs, it is reasonable to use the renal vein as the upper border of the radiation therapy field for patients treated with prophylactic EFI. The node CTV expansion from the vessel should be smaller in the right para-caval region than in the LLPA region. More clinical trials are needed on prophylactic EFI in cervical cancer.

\section{AUTHOR CONTRIBUTIONS}

$\mathrm{KH}$ and FZ contributed to the conception of the study. WW and YZ wrote the first draft of the manuscript. DW contributed to the review of literatures. All authors contributed to the manuscript revision, read, and approved the submitted version.

\section{FUNDING}

This work was supported by the National Natural Science Foundation of China (grant number U19A2064) and the National Key Technologies Research and Development Program of China (grant number 2016YFC0105207).

cervical cancer. Gynecol Oncol. (2017) 144:40-5. doi: 10.1016/j.ygyno.2016. 11.011

4. Berman ML, Keys H, Creasman W, DiSaia P, Bundy B, Blessing J. Survival and patterns of recurrence in cervical cancer metastatic to periaortic lymph nodes (a Gynecologic Oncology Group study). Gynecol Oncol. (1984) 19:8-16. doi: 10.1016/0090-8258(84) 90151-3

5. De Cuypere M, Lovinfosse P, Goffin F, Gennigens C, Rovira R, Duch J, et al. Added value of para-aortic surgical staging compared to (18)F-FDG PET/CT on the external beam radiation field for patients with locally advanced cervical 
cancer: an ONCO-GF study. Eur J Surg Oncol. (2019) 46:883-7. doi: 10.1016/ j.ejso.2019.11.496

6. Bhatla N, Aoki D, Sharma DN, Sankaranarayanan R. Cancer of the cervix uteri. Int J Gynaecol Obstet. (2018) 143(Suppl. 2):22-36. doi: 10.1002/ijgo.12611

7. Small W Jr., Winter K, Levenback C, Iyer R, Gaffney D, Asbell S, et al. Extended-field irradiation and intracavitary brachytherapy combined with cisplatin chemotherapy for cervical cancer with positive para-aortic or high common iliac lymph nodes: results of ARM 1 of RTOG 0116. Int J Radiat Oncol Biol Phys. (2007) 68:1081-7. doi: 10.1016/j.ijrobp.2007.01.026

8. Liu X, Wang W, Meng Q, Zhang F, Hu K. Extended-field intensity-modulated radiation therapy combined with concurrent chemotherapy for cervical cancer with para-aortic lymph nodes metastasis. Jpn J Clin Oncol. (2018) 49:263-9. doi: 10.1093/jjco/hyy184

9. Tan LT, Potter R, Sturdza A, Fokdal L, Haie-Meder C, Schmid M, et al. Change in patterns of failure after image-guided brachytherapy for cervical cancer: analysis from the RetroEMBRACE study. Int J Radiat Oncol Biol Phys. (2019) 104:895-902. doi: 10.1016/j.ijrobp.2019.03.038

10. Nomden CN, Potter R, de Leeuw AAC, Tanderup K, Lindegaard JC, Schmid MP, et al. Nodal failure after chemo-radiation and MRI guided brachytherapy in cervical cancer: patterns of failure in the EMBRACE study cohort. Radiother Oncol. (2019) 134:185-90. doi: 10.1016/j.radonc.2019.02.007

11. Huang EY, Wang CJ, Chen HC, Fang FM, Huang YJ, Wang CY, et al. Multivariate analysis of para-aortic lymph node recurrence after definitive radiotherapy for stage IB-IVA squamous cell carcinoma of uterine cervix. Int $J$ Radiat Oncol Biol Phys. (2008) 72:834-42. doi: 10.1016/j.ijrobp.2008.01.035

12. Lai C-H, Huang K-G, Hong J-H, Lee C-L, Chou H-H, Chang T-C, et al. Randomized trial of surgical staging (extraperitoneal or laparoscopic) versus clinical staging in locally advanced cervical cancer?. Gynecol Oncol. (2003) 89:160-7. doi: 10.1016/s0090-8258(03)00064-7

13. Yang J, Delara R, Magrina J, Magtibay P, Yi J, Langstraat C, et al. Comparing survival outcomes between surgical and radiographic lymph node assessment in locally advanced cervical cancer: a propensity score-matched analysis. Gynecol Oncol. (2020) 156:320-7. doi: 10.1016/j.ygyno.2019.12.009

14. Gonzalez-Benitez C, Salas P, Grabowski JP, Hernandez A, De Santiago J, Zapardiel I. Lack of survival benefit of para-aortic lymphadenectomy in advanced cervical cancer. Gynecol Obstet Invest. (2019) 84:407-11. doi: 10. $1159 / 000497350$

15. Gold MA, Tian C, Whitney CW, Rose PG, Lanciano R. Surgical versus radiographic determination of para-aortic lymph node metastases before chemoradiation for locally advanced cervical carcinoma: a gynecologic oncology group study. Cancer. (2008) 112:1954-63. doi: 10.1002/cncr.23400

16. Marnitz S, Martus P, Kohler C, Stromberger C, Asse E, Mallmann P, et al. Role of surgical versus clinical staging in chemoradiated FIGO stage IIB-IVA cervical cancer patients-acute toxicity and treatment quality of the Uterus11 Multicenter Phase III intergroup trial of the german radiation oncology group and the gynecologic cancer group. Int J Radiat Oncol Biol Phys. (2016) 94:243-53. doi: 10.1016/j.ijrobp.2015.10.027

17. Liu B, Gao S, Li S. A comprehensive comparison of CT, MRI, positron emission tomography or positron emission tomography/CT, and diffusion weighted imaging-MRI for detecting the lymph nodes metastases in patients with cervical cancer: a meta-analysis based on 67 studies. Gynecol Obstet Invest. (2017) 82:209-22. doi: 10.1159/000456006

18. Yu W, Kou C, Bai W, Yu X, Duan R, Zhu B, et al. The diagnostic performance of PET/CT scans for the detection of para-aortic metastatic lymph nodes in patients with cervical cancer: a meta-analysis. PLoS One. (2019) 14:e0220080. doi: 10.1371/journal.pone.0220080

19. Martinez A, Voglimacci M, Lusque A, Ducassou A, Gladieff L, Dupuis N, et al. Tumour and pelvic lymph node metabolic activity on FDG-PET/CT to stratify patients for para-aortic surgical staging in locally advanced cervical cancer. Eur J Nucl Med Mol Imaging. (2020) 47:1252-60. doi: 10.1007/s00259-01904659-z

20. Uzan C, Souadka A, Gouy S, Debaere T, Duclos J, Lumbroso J, et al. Analysis of morbidity and clinical implications of laparoscopic para-aortic lymphadenectomy in a continuous series of 98 patients with advancedstage cervical cancer and negative PET-CT imaging in the para-aortic area. Oncologist. (2011) 16:1021-7. doi: 10.1634/theoncologist.2011-0007

21. Ramirez PT, Jhingran A, Macapinlac HA, Euscher ED, Munsell MF, Coleman $\mathrm{RL}$, et al. Laparoscopic extraperitoneal para-aortic lymphadenectomy in locally advanced cervical cancer: a prospective correlation of surgical findings with positron emission tomography/computed tomography findings. Cancer. (2011) 117:1928-34. doi: 10.1002/cncr.25739

22. Rotman M, Pajak TF, Choi K, Clery M, Marcial V, Grigsby PW, et al. Prophylactic extended-field irradiation of para-aortic lymph nodes in stages IIB and bulky IB and IIA cervical carcinomas. Ten-year treatment results of RTOG 79-20. JAMA. (1995) 274:387-93. doi: 10.1001/jama.1995. 03530050035029

23. Haie C, Pejovic MH, Gerbaulet A, Horiot JC, Pourquier H, Delouche J, et al. Is prophylactic para-aortic irradiation worthwhile in the treatment of advanced cervical carcinoma? Results of a controlled clinical trial of the EORTC radiotherapy group. Radiother Oncol. (1988) 11:101-12. doi: 10.1016/ 0167-8140(88)90245-9

24. Chatani M, Matayoshi Y, Masaki N, Narumi Y, Teshima T, Inoue T. Prophylactic irradiation of para-aortic lymph nodes in carcinoma of the uterine cervix. A prospective randomized study. Strahlenther Onkol. (1995) 171:655-60.

25. Thamronganantasakul K, Supakalin N, Kietpeerakool C, Pattanittum P, Lumbiganon P. Extended-field radiotherapy for locally advanced cervical cancer. Cochrane Database Syst Rev. (2018) 10:CD012301. doi: 10.1002/ 14651858.CD012301.pub2

26. Peters WA III, Liu PY, Barrett RJ II, Stock RJ, Monk BJ, Berek JS, et al. Concurrent chemotherapy and pelvic radiation therapy compared with pelvic radiation therapy alone as adjuvant therapy after radical surgery in highrisk early-stage cancer of the cervix. J Clin Oncol. (2000) 18:1606-13. doi: 10.1200/jco.2000.18.8.1606

27. Whitney CW, Sause W, Bundy BN, Malfetano JH, Hannigan EV, Fowler WC Jr., et al. Randomized comparison of fluorouracil plus cisplatin versus hydroxyurea as an adjunct to radiation therapy in stage IIB-IVA carcinoma of the cervix with negative para-aortic lymph nodes: a Gynecologic Oncology Group and Southwest Oncology Group study. J Clin Oncol. (1999) 17:1339-48. doi: 10.1200/JCO.1999.17.5.1339

28. Rose PG, Bundy BN, Watkins EB, Thigpen JT, Deppe G, Maiman MA, et al. Concurrent cisplatin-based radiotherapy and chemotherapy for locally advanced cervical cancer. N Engl J Med. (1999) 340:1144-53. doi: 10.1056/ NEJM199904153401502

29. Keys HM, Bundy BN, Stehman FB, Muderspach LI, Chafe WE, Suggs CL III, et al. Cisplatin, radiation, and adjuvant hysterectomy compared with radiation and adjuvant hysterectomy for bulky stage IB cervical carcinoma. N Engl J Med. (1999) 340:1154-61. doi: 10.1056/NEJM199904153401503

30. Morris M, Eifel PJ, Lu J, Grigsby PW, Levenback C, Stevens RE, et al. Pelvic radiation with concurrent chemotherapy compared with pelvic and para-aortic radiation for high-risk cervical cancer. N Engl J Med. (1999) 340:1137-43. doi: 10.1056/NEJM199904153401501

31. Eifel PJ, Winter K, Morris M, Levenback C, Grigsby PW, Cooper J, et al. Pelvic irradiation with concurrent chemotherapy versus pelvic and paraaortic irradiation for high-risk cervical cancer: an update of radiation therapy oncology group trial (RTOG) 90-01. J Clin Oncol. (2004) 22:872-80. doi: 10.1200/JCO.2004.07.197

32. Asiri MA, Tunio MA, Mohamed R, Bayoumi Y, Alhadab A, Saleh RM, et al. Is extended-field concurrent chemoradiation an option for radiologic negative paraaortic lymph node, locally advanced cervical cancer? Cancer Manag Res. (2014) 6:339-48. doi: 10.2147/CMAR.S68262

33. Lee J, Lin JB, Chang CL, Jan YT, Sun FJ, Wu MH, et al. Prophylactic lower para-aortic irradiation using intensity-modulated radiotherapy mitigates the risk of para-aortic recurrence in locally advanced cervical cancer: a 10-year institutional experience. Gynecol Oncol. (2017) 146:20-6. doi: 10.1016/j.ygyno. 2017.04.016

34. Wang W, Liu X, Meng Q, Zhang F, Hu K. Prophylactic extendedfield irradiation for patients with cervical cancer treated with concurrent chemoradiotherapy: a propensity-score matching analysis. Int J Gynecol Cancer. (2018) 28:1584-91. doi: 10.1097/IGC.0000000000001344

35. Ouyang Y, Wang Y, Chen K, Cao X, Zeng Y. Clinical outcome of extendedfield irradiation vs. pelvic irradiation using intensity-modulated radiotherapy for cervical cancer. Oncol Lett. (2017) 14:7069-76. doi: 10.3892/ol.2017.7077

36. Oh J, Seol KH, Lee HJ, Choi YS, Park JY, Bae JY. Prophylactic extendedfield irradiation with concurrent chemotherapy for pelvic lymph node-positive cervical cancer. Radiat Oncol J. (2017) 35:349-58. doi: 10.3857/roj.2017.00367 
37. Park SG, Kim JH, Oh YK, Byun SJ, Kim MY, Kwon SH, et al. Is prophylactic irradiation to para-aortic lymph nodes in locally advanced cervical cancer necessary? Cancer Res Treat. (2014) 46:374-82. doi: 10.4143/crt.2013.084

38. Yap ML, Cuartero J, Yan J, Pintilie M, Fyles A, Levin W, et al. The role of elective para-aortic lymph node irradiation in patients with locally advanced cervical cancer. Clin Oncol (R Coll Radiol). (2014) 26:797-803. doi: 10.1016/j. clon.2014.08.008

39. ClinicalTrials.gov. Prophylactic Irradiation of the Para-Aortic Lymph Nodes in Locally Advanced Uterine Cervical Cancer. (2019). Available online at: https://clinicaltrials.gov/ct2/show/NCT00980759?term=NCT+00980759\& draw=2\&rank=1 (accessed March 20, 2019).

40. Kim JH, Kim JY, Yoon MS, Kim YS, Lee JH, Kim HJ, et al. Prophylactic irradiation of para-aortic lymph nodes for patients with locally advanced cervical cancers with and without high CA9 expression (KROG 07-01): a randomized, open-label, multicenter, phase 2 trial. Radiother Oncol. (2016) 120:383-9. doi: 10.1016/j.radonc.2016.04.009

41. ClinicalTrials.gov. Pelvic RT Versus Prophylactic Extended-field RT in Patients With Cervical Cancer. (2019). Available online at: https://clinicaltrials.gov/ct2/ show/NCT03955367?term=Hu+Ke\&draw=2\&rank=1. (accessed March 20, 2019).

42. ClinicalTrials.gov. Prophylactic Irradiation of the Para-Aortic Lymph Nodes and Supraclavicular Lymph Nodes in Locally Advanced Uterine Cervical Cancer. (2019). Available online at: https://clinicaltrials.gov/ct2/show/ NCT01063387? cond=NCT $+01063387 \&$ draw $=2 \&$ rank=1 (accessed March 20, 2019).

43. ClinicalTrials.gov. Phase II Clinical Trial of Extended field IMRT Chemoradiotherapy or pelvic IMRT Chemoradiotherapy Followed by Operation in Patients with Multiple Pelvic Lymphanode Metastasis. (2019). Available online at: http://www.chictr.org.cn/showproj.aspx?proj=9816 (accessed March 20, 2019)

44. ClinicalTrials.gov. A Phase III Trial of Intensity Modulated Radiation Therapy for pelvic and/or Para-Aortic Nodes and Intra-Cavitary Brachytherapy Combined with Chemotherapy for Stage IIb-IVa Cervical cancer. (2019). Available online at: http://www.chictr.org.cn/showproj.aspx?proj=13712 (accessed March 20, 2019)

45. Potter R, Tanderup K, Kirisits C, de Leeuw A, Kirchheiner K, Nout R, et al. The EMBRACE II study: the outcome and prospect of two decades of evolution within the GEC-ESTRO GYN working group and the EMBRACE studies. Clin Transl Radiat Oncol. (2018) 9:48-60. doi: 10.1016/j.ctro.2018.01.001

46. Grigsby PW, Heydon K, Mutch DG, Kim RY, Eifel P. Long-term followup of RTOG 92-10: cervical cancer with positive para-aortic lymph nodes. Int J Radiat Oncol Biol Phys. (2001) 51:982-7. doi: 10.1016/s0360-3016(01) 01723-0

47. Lee J, Lin JB, Chang CL, Sun FJ, Wu MH, Jan YT, et al. Impact of para-aortic recurrence risk-guided intensity-modulated radiotherapy in locally advanced cervical cancer with positive pelvic lymph nodes. Gynecol Oncol. (2018) 148:291-8. doi: 10.1016/j.ygyno.2017.12.003

48. Lin Y, Chen K, Lu Z, Zhao L, Tao Y, Ouyang Y, et al. Intensity-modulated radiation therapy for definitive treatment of cervical cancer: a meta-analysis. Radiat Oncol. (2018) 13:177. doi: 10.1186/s13014-018-1126-7

49. Naik A, Gurjar OP, Gupta KL, Singh K, Nag P, Bhandari V. Comparison of dosimetric parameters and acute toxicity of intensity-modulated and threedimensional radiotherapy in patients with cervix carcinoma: a randomized prospective study. Cancer Radiother. (2016) 20:370-6. doi: 10.1016/j.canrad. 2016.05.011

50. Yu C, Zhu W, Ji Y, Guo J, Pan P, Han J, et al. A comparative study of intensity-modulated radiotherapy and standard radiation field with concurrent chemotherapy for local advanced cervical cancer. Eur J Gynaecol Oncol. (2015) 36:278-82.

51. Gandhi AK, Sharma DN, Rath GK, Julka PK, Subramani V, Sharma S, et al. Early clinical outcomes and toxicity of intensity modulated versus conventional pelvic radiation therapy for locally advanced cervix carcinoma: a prospective randomized study. Int J Radiat Oncol Biol Phys. (2013) 87:542-8. doi: 10.1016/j.ijrobp.2013.06.2059

52. Chino J, Annunziata CM, Beriwal S, Bradfield L, Erickson BA, Fields EC, et al. Radiation therapy for cervical cancer: executive summary of an ASTRO clinical practice guideline. Pract Radiat Oncol. (2020) 10:220-34. doi: 10.1016/ j.prro.2020.04.002
53. Wang W, Zhang $\mathrm{F}, \mathrm{Hu} \mathrm{K}$, Hou $\mathrm{X}$. Image-guided, intensity-modulated radiation therapy in definitive radiotherapy for 1433 patients with cervical cancer. Gynecol Oncol. (2018) 151:444-8. doi: 10.1016/j.ygyno.2018.09.024

54. Vandeperre A, Van Limbergen E, Leunen K, Moerman P, Amant F, Vergote I. Para-aortic lymph node metastases in locally advanced cervical cancer: comparison between surgical staging and imaging. Gynecol Oncol. (2015) 138:299-303. doi: 10.1016/j.ygyno.2015.05.021

55. Han X, Wen H, Ju X, Chen X, Ke G, Zhou Y, et al. Predictive factors of para-aortic lymph nodes metastasis in cervical cancer patients: a retrospective analysis based on 723 para-aortic lymphadenectomy cases. Oncotarget. (2017) 8:51840-7. doi: 10.18632/oncotarget.16025

56. Wang W, Liu X, Meng Q, Zhang F, Hu K. Nomogram for predicting paraaortic lymph node metastases in patients with cervical cancer. Arch Gynecol Obstet. (2018) 298:381-8. doi: 10.1007/s00404-018-4829-y

57. Sapienza LG, Gomes MJL, Calsavara VF, Leitao MM Jr., Baiocchi G. Does para-aortic irradiation reduce the risk of distant metastasis in advanced cervical cancer? A systematic review and meta-analysis of randomized clinical trials. Gynecol Oncol. (2017) 144:312-7. doi: 10.1016/j.ygyno.2016.11.044

58. Liu X, Meng Q, Wang W, Zhou Z, Zhang F, Hu K. Predictors of distant metastasis in patients with cervical cancer treated with definitive radiotherapy. J Cancer. (2019) 10:3967-74. doi: 10.7150/jca.31538

59. Je HU, Han S, Kim YS, Nam JH, Kim HJ, Kim JW, et al. A nomogram predicting the risks of distant metastasis following postoperative radiotherapy for uterine cervical carcinoma: a Korean radiation oncology group study (KROG 12-08). Radiother Oncol. (2014) 111:437-41. doi: 10.1016/j.radonc. 2014.03.025

60. Kang S, Nam BH, Park JY, Seo SS, Ryu SY, Kim JW, et al. Risk assessment tool for distant recurrence after platinum-based concurrent chemoradiation in patients with locally advanced cervical cancer: a Korean gynecologic oncology group study. J Clin Oncol. (2012) 30:2369-74. doi: 10.1200/JCO.2011.37.5923

61. Wang W, Liu X, Meng Q, Zhang F, Hu K. Nomograms predicting survival and patterns of failure in patients with cervical cancer treated with concurrent chemoradiotherapy: a special focus on lymph nodes metastases. PLoS One. (2019) 14:e0214498. doi: 10.1371/journal.pone.0214498

62. Meng Q, Liu X, Wang W, Hou X, Lian X, Sun S, et al. Evaluation of the efficacy of prophylactic extended field irradiation in the concomitant chemoradiotherapy treatment of locally advanced cervical cancer, stage IIIB in the 2018 FIGO classification. Radiat Oncol. (2019) 14:228. doi: 10.1186/ s13014-019-1431-9

63. National Comprehensive Cancer Network(NCCN). Clinical Practice Guidelines in Oncology. Cervical Cancer.Version 3.2019. (2019). Available online at: https://www.nccn.org/professionals/physician_gls/default.aspx\# cervical (accessed March 13, 2019)

64. Jurgenliemk-Schulz IM, Beriwal S, de Leeuw AAC, Lindegaard JC, Nomden $\mathrm{CN}$, Potter R, et al. Management of nodal disease in advanced cervical cancer. Semin Radiat Oncol. (2019) 29:158-65. doi: 10.1016/j.semradonc.2018.11.002

65. Hu K, Wang W, Liu X, Meng Q, Zhang F. Comparison of treatment outcomes between squamous cell carcinoma and adenocarcinoma of cervix after definitive radiotherapy or concurrent chemoradiotherapy. Radiat Oncol. (2018) 13:249. doi: 10.1186/s13014-018-1197-5

66. Choi KH, Lee SW, Yu M, Jeong S, Lee JW, Lee JH. Significance of elevated SCC-Ag level on tumor recurrence and patient survival in patients with squamous-cell carcinoma of uterine cervix following definitive chemoradiotherapy: a multi-institutional analysis. J Gynecol Oncol. (2019) 30:e1. doi: 10.3802/jgo.2019.30.e1

67. Keenan LG, Rock K, Azmi A, Salib O, Gillham C, McArdle O. An atlas to aid delineation of para-aortic lymph node region in cervical cancer: design and validation of contouring guidelines. Radiother Oncol. (2018) 127:417-22. doi: 10.1016/j.radonc.2018.02.013

68. Takiar V, Fontanilla HP, Eifel PJ, Jhingran A, Kelly P, Iyer RB, et al. Anatomic distribution of fluorodeoxyglucose-avid para-aortic lymph nodes in patients with cervical cancer. Int J Radiat Oncol Biol Phys. (2013) 85:1045-50. doi: 10.1016/j.ijrobp.2012.11.032

69. Chao KS, Lin M. Lymphangiogram-assisted lymph node target delineation for patients with gynecologic malignancies. Int J Radiat Oncol Biol Phys. (2002) 54:1147-52. doi: 10.1016/s0360-3016(02)03040-7

70. Kabolizadeh P, Fulay S, Beriwal S. Are radiation therapy oncology group para-aortic contouring guidelines for pancreatic neoplasm applicable to other 
malignancies-assessment of nodal distribution in gynecological malignancies. Int J Radiat Oncol Biol Phys. (2013) 87:106-10. doi: 10.1016/j.ijrobp.2013.05. 034

71. Yang B, Liu X, Hu K, Qiu J, Zhang F, Hou X, et al. Reduction of dose to duodenum with a refined delineation method of Para-aortic region in patients with locally advanced cervical Cancer receiving prophylactic extended-field radiotherapy. Radiat Oncol. (2019) 14:196. doi: 10.1186/s13014-019-1398-6

72. Kohler C, Mustea A, Marnitz S, Schneider A, Chiantera V, Ulrich U, et al. Perioperative morbidity and rate of upstaging after laparoscopic staging for patients with locally advanced cervical cancer: results of a prospective randomized trial. Am J Obstet Gynecol. (2015) 213:503.e1-7. doi: 10.1016/j. ajog.2015.05.026

73. Mezquita G, Muruzabal JC, Perez B, Aguirre S, Villafranca E, Jurado M. Para-aortic plus pelvic lymphadenectomy in locally advanced cervical cancer: a single institutional experience. Eur J Obstet Gynecol Reprod Biol. (2019) 236:79-83. doi: 10.1016/j.ejogrb.2019.02.033

74. Gouy S, Morice P, Narducci F, Uzan C, Gilmore J, Kolesnikov-Gauthier H, et al. Nodal-staging surgery for locally advanced cervical cancer in the era of PET. Lancet Oncol. (2012) 13:e212-20. doi: 10.1016/S1470-2045(12)70011-6
75. Becker JH, Verheijen R. Locally advanced cervix cancer: staging by scan or by surgery? BJOG. (2017) 124:1096. doi: 10.1111/1471-0528. 14596

76. Lanciano R, Calkins A, Bundy BN, Parham G, Lucci JA III, Moore DH, et al. Randomized comparison of weekly cisplatin or protracted venous infusion of fluorouracil in combination with pelvic radiation in advanced cervix cancer: a gynecologic oncology group study. J Clin Oncol. (2005) 23:8289-95. doi: 10.1200/JCO.2004.00.0497

Conflict of Interest: The authors declare that the research was conducted in the absence of any commercial or financial relationships that could be construed as a potential conflict of interest.

Copyright $\odot 2020$ Wang, Zhou, Wang, Hu and Zhang. This is an open-access article distributed under the terms of the Creative Commons Attribution License (CC BY). The use, distribution or reproduction in other forums is permitted, provided the original author(s) and the copyright owner(s) are credited and that the original publication in this journal is cited, in accordance with accepted academic practice. No use, distribution or reproduction is permitted which does not comply with these terms. 